\title{
Turing Computations on Ordinals
}

\author{
Peter Koepke \\ University of Bonn
}

October 11, 2018

\begin{abstract}
We define the notion of ordinal computability by generalizing standard TURING computability on tapes of length $\omega$ to computations on tapes of arbitrary ordinal length. We show that a set of ordinals is ordinal computable from a finite set of ordinal parameters if and only if it is an element of GöDEL's constructible universe $L$. This characterization can be used to prove the generalized continuum hypothesis in $L$.
\end{abstract}

\section{Introduction.}

A standard TURING computation may be visualized as a time-like sequence of elementary read-write-move operations carried out by one or more "heads" on "tapes". The sequence of actions is determined by the initial tape contents and by a finite TURING program. The specific choice of alphabet, operations and tapes may influence the time or space complexity of calculations; by the Church-TuRING thesis, however, the associated notion of TURING computability is not affected. So we may assume that TURING machines act on tapes whose cells are indexed by the set $\omega(=\mathbb{N})$ of natural numbers $0,1, \ldots$ and contain 0 's or 1's.

\begin{tabular}{|c|c|c|c|c|c|c|c|c|c|c|c|}
\hline & & & $\mathrm{S}$ & $\mathrm{P}$ & $\mathrm{A}$ & $\mathrm{C}$ & $\mathrm{E}$ & & & & \\
\hline & & 0 & 1 & 2 & 3 & 4 & 5 & 6 & 7 & $\ldots$ & $\ldots$ \\
\hline & 0 & $\underline{1}$ & 0 & 0 & 1 & 1 & 1 & 0 & 0 & 0 & 0 \\
\hline & 1 & 0 & $\underline{0}$ & 0 & 1 & 1 & 1 & 0 & 0 & & \\
\hline $\mathrm{T}$ & 2 & 0 & 0 & $\underline{0}$ & 1 & 1 & 1 & 0 & 0 & & \\
\hline $\mathrm{I}$ & 3 & 0 & $\underline{0}$ & 1 & 1 & 1 & 1 & 0 & 0 & & \\
\hline $\mathrm{M}$ & 4 & 0 & 1 & $\underline{1}$ & 1 & 1 & 1 & 0 & 0 & & \\
\hline $\mathrm{E}$ & $:$ & & & & & & & & & & \\
\hline & $n$ & 1 & 1 & 1 & 1 & $\underline{0}$ & 1 & 1 & 1 & & \\
\hline & $n+1$ & 1 & 1 & 1 & 1 & 1 & $\underline{1}$ & 1 & 1 & & \\
\hline & $\vdots$ & & & & & & & & & & \\
\hline
\end{tabular}

A standard TURING computation. Head positions are indicated by underlining. 
An obvious generalization from the perspective of transfinite ordinal theory is to extend TURING calculations to tapes whose cells are indexed by the class Ord of all ordinal numbers. Calculations will become (infinite) sequences of elementary tape operations indexed by ordinals which may be viewed as instances of time. For successor ordinals (or times) calculations will basically be defined as for standard TURING machines. At limit ordinals we define the tape contents, program states and head positions by appropriate limit operations which may be viewed as inferior limits.

\begin{tabular}{|c|c|c|c|c|c|c|c|c|c|c|c|c|c|c|c|}
\hline & & $\mathrm{O}$ & $\mathrm{r}$ & $\mathrm{d}$ & $\mathrm{i}$ & $\mathrm{n}$ & $\mathrm{a}$ & $\mathrm{l}$ & & $\mathrm{S}$ & $\mathrm{p}$ & $\mathrm{a}$ & $\mathrm{c}$ & $\mathrm{e}$ & $\ldots$ \\
\hline & & 0 & 1 & 2 & 3 & 4 & 5 & 6 & 7 & $\ldots$ & $\ldots$ & $\omega$ & $\ldots$ & $\alpha$ & $\ldots$ \\
\hline $\mathrm{O}$ & 0 & $\underline{1}$ & 1 & 0 & 1 & 0 & 0 & 1 & 1 & $\ldots$ & $\ldots$ & 1 & $\ldots$ & 1 & 0 \\
\hline $\mathrm{r}$ & 1 & 0 & $\underline{1}$ & 0 & 1 & 0 & 0 & 1 & 1 & & & 1 & & & \\
\hline $\mathrm{d}$ & 2 & 0 & 0 & $\underline{0}$ & 1 & 0 & 0 & 1 & 1 & & & 1 & & & \\
\hline $\mathrm{i}$ & 3 & 0 & $\underline{0}$ & 0 & 1 & 0 & 0 & 1 & 1 & & & 1 & & & \\
\hline $\mathrm{n}$ & 4 & 0 & 0 & $\underline{0}$ & 0 & 0 & 0 & 1 & 1 & & & 1 & & & \\
\hline $\mathrm{a}$ & $\vdots$ & & & & & & & & & & & & & & \\
\hline $\mathrm{l}$ & $\mathrm{n}$ & 1 & 1 & 1 & 1 & 0 & 1 & 0 & $\underline{1}$ & & & 1 & & & \\
\hline & $\mathrm{n}+1$ & 1 & 1 & 1 & 1 & 1 & 1 & $\underline{0}$ & 1 & & & 1 & & & \\
\hline $\mathrm{T}$ & $\vdots$ & $\vdots$ & $\vdots$ & $\vdots$ & $\vdots$ & $\vdots$ & & & & & & & & & \\
\hline $\mathrm{i}$ & $\omega$ & 0 & 0 & 1 & 0 & 0 & 0 & 1 & 1 & $\ldots$ & $\ldots$ & $\underline{1}$ & & & \\
\hline $\mathrm{m}$ & $\omega+1$ & $\underline{0}$ & 0 & 1 & 0 & 0 & 0 & 1 & 1 & & & 0 & & & \\
\hline $\mathrm{e}$ & $\vdots$ & & & & & & & & & & & & & & \\
\hline$\vdots$ & $\theta$ & 1 & 0 & 0 & 1 & 1 & 1 & 1 & 0 & $\ldots$ & $\ldots$ & $\ldots$ & $\ldots$ & $\underline{0}$ & $\ldots$ \\
\hline$\vdots$ & $\vdots$ & & & $\vdots$ & & & $\vdots$ & & & $\vdots$ & $\vdots$ & & & & \\
\hline$\vdots$ & $\vdots$ & & & & & & & & & & & & & & \\
\hline
\end{tabular}

An ordinal computation.

The corresponding notion of ordinal computability obviously extends TURING computability. By the CHURCH-TURING thesis many operations on natural numbers are ordinal computable. The ordinal arithmetical operations (addition, multiplication, exponentiation) and other basic operations on ordinals are also ordinal computable.

Indeed, the recursive properties of the family of ordinal computable functions are so strong that the bounded truth predicate

$$
\left\{(\alpha, \varphi, \vec{x}) \mid \alpha \in \text { Ord, } \varphi \text { an } \in \text {-formula, } \vec{x} \in L_{\alpha}, L_{\alpha} \vDash \varphi(\vec{x})\right\}
$$

for GÖDEL's constructible hierarchy $L=\bigcup_{\alpha \in \text { Ord }} L_{\alpha}$ is ordinal computable given some appropriate coding. As a corollary we obtain the main result characterizing ordinal computability:

Theorem 1 A set $x \subseteq$ Ord is ordinal computable from finitely many ordinal parameters if and only if $x \in L$. 
The implication from left to right will be immediate from the set-theoretical absoluteness of ordinal computations. The converse requires a careful analysis of the iterative definition of the constructible hierarchy to show that the iteration can be carried out by an ordinal TURING machine.

This theorem may be viewed as an analogue of the CHURCH-TURING thesis: ordinal computability defines a natural and absolute class of sets, and it is stable with respect to technical variations in its definition.

Theories of transfinite computations which extend TURING computability have been proposed and studied for some time. Higher recursion theory as described in the monograph [6] of GERALD SACKS culminates in E-recursion which defines a computational result $\{e\}(x)$ for programs $e$ built from basic set functions applied to arbitrary sets $x$. The relation of $E$-computability to constructibility is analogous to the statement of Theorem 1. In computer science various infinitary machines like BÜCHI automata [1 have been defined yielding important applications. The novelty here is in keeping with the original TURING idea of reading and writing on an unrestricted tape while interpreting unrestrictedness in the widest sense as set-theoretical unboundedness.

Our work was inspired by the infinite time TURING machines introduced by Joel D. Hamkins, JefF Kidder and Andy Lewis 4]. Infinite time Turing machines use standard tapes indexed by natural numbers but allow infinite computation sequences. At limit times, tape contents are defined as inferior limits of previous contents. Inputs and outputs are characteristic functions on the set $\omega$ of all natural numbers and may thus be viewed as real numbers. The theory of infinite time TURING machines is naturally related to definability theory over the structure $(\mathbb{R}, \ldots)$, i.e., to descriptive set theory. In the case of tapes of arbitrary ordinal length one is lead to consider a theory of arbitrarily iterated definitions, i.e., constructibility theory.

\section{Ordinal Turing Machines}

We give an intuitive description of ordinal computations which will subsequently be formalized. Consider a tape of ordertype Ord, i.e., a sequence indexed by the class Ord of all ordinals. The cells of the tape can be identified with the ordinals, every cell can contain a 0 or a 1 where 0 is the default state. A readwrite head moves on the tape, starting at cell 0 . The computation is steered by a program which consists of a finite sequence of commands indexed by natural numbers. The indices of the commands can be seen as states of the machine.

A computation of the machine is a sequence of machine configurations which are indexed by ordinal "times" $0,1,2, \ldots, \omega, \omega+1, \ldots$ At time $t$ the read-write head reads the content of the cell at its position. According to the content and the present machine state the head writes a "0" or a " 1 " and then moves to the right or to the left. Also the machine changes into a new program state.

So far we have described the computation rules of finitary TURING machines. Ordinal computations require the specification of the behaviour at limit ordinals; we shall base the limit rules on simple limit operations. 
Assume that at time $t$ the head position is $H(t)$. After a move-right command we put $H(t+1)=H(t)+1$. After a move-left command we move one cell to the left if possible and otherwise, if $H(t)$ is a limit ordinal or 0 , jump to the default position 0 :

$$
H(t+1)=\left\{\begin{array}{l}
H(t)-1, \text { if } H(t) \text { is a successor ordinal; } \\
0, \text { else. }
\end{array}\right.
$$

The definition of $H(t)$ for $t$ a limit ordinal will be given later.

At time $t$ the tape will be identified with a tape content

$$
T(t)=\left(T(t)_{0}, T(t)_{1}, \ldots, T(t)_{\omega}, T(t)_{\omega+1}, \ldots\right)
$$

which is a sequence of cell contents $T(t)_{\alpha} \in\{0,1\}$. It is determined by previous write operations. For limit times $t$ the content $T(t)_{\alpha}$ of the $\alpha$-th cell is determined as follows: if the cell content $T(s)_{\alpha}$ stabilizes at a constant value as $s$ approaches $t$ we let $T(t)_{\alpha}$ be that value; otherwise we take the default value $T(t)_{\alpha}=0$. Formally this is an inferior limit:

$$
T(t)_{\alpha}=\liminf _{s \rightarrow t} T(s)_{\alpha} .
$$

A liminf rule will also be used for the program state and the head location at limit times. Let $S(t)$ be the program state at time $t$. For limit times $t$ set

$$
S(t)=\liminf _{s \rightarrow t} S(s)
$$

Finally the head position $H(t)$ for limit times $t$ is

$$
H(t)=\liminf _{s \rightarrow t, S(s)=S(t)} H(s) .
$$

The definitions of $S(t)$ and $H(t)$ can be motivated as follows. Since a Turing program is finite its execution will lead to some (complex) looping structure involving loops, subloops and so forth. This can be presented by pseudo code like:

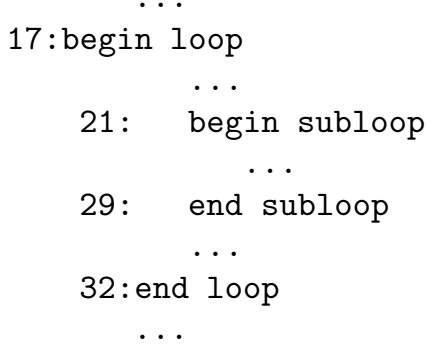

Assume that for times $s \rightarrow t$ the loop $(17-32)$ with its subloop $(21-29)$ is traversed cofinally often. Then at limit time $t$ it is natural to put the machine 
at the start of the "main loop". Assuming that the lines of the program are enumerated in increasing order this corresponds to the lim inf rule

$$
S(t)=\liminf _{s \rightarrow t} S(s) .
$$

The canonical head location $H(t)$ is then determined as the inferior limit of all head locations when the program is at the start of the "main loop". If the head is for example moving linearly towards a limit location, say $H\left(s_{0}+i\right)=h_{0}+i$ for $i<\lambda$, we will have $H\left(s_{0}+\lambda\right)=h_{0}+\lambda$. Note that the limit behaviour of the head position is defined differently for infinite time TURING machines which do

not possess limit positions on the tape; there the head simply falls back to 0 at limit times.

The above intuitions are formalized as follows.

\section{Definition 1}

a) A command is a 5-tuple $C=\left(s, c, c^{\prime}, m, s^{\prime}\right)$ where $s, s^{\prime} \in \omega$ and $c, c^{\prime}, m \in$ $\{0,1\}$; the natural number $s$ is the state of the command $C$. The intention of the command $C$ is that if the machine is in state $s$ and reads the symbol $c$ under its read-write head, then it writes the symbol $c^{\prime}$, moves the head left if $m=0$ or right if $m=1$, and goes into state $s^{\prime}$. States correspond to the "line numbers" of some programming languages.

b) A program is a finite set $P$ of commands satisfying the following structural conditions:

i. If $\left(s, c, c^{\prime}, m, s^{\prime}\right) \in P$ then there is $\left(s, d, d^{\prime}, n, t^{\prime}\right) \in P$ with $c \neq d$; thus in state $s$ the machine can react to reading a " 0 " as well as to reading a "1".

ii. If $\left(s, c, c^{\prime}, m, s^{\prime}\right) \in P$ and $\left(s, c, c^{\prime \prime}, m^{\prime}, s^{\prime \prime}\right) \in P$ then $c^{\prime}=c^{\prime \prime}, m=m^{\prime}, s^{\prime}=$ $s^{\prime \prime}$; this means that the course of the computation is completely determined by the sequence of program states and the initial cell contents.

c) For a program $P$ let

$$
\operatorname{states}(P)=\left\{s \mid\left(s, c, c^{\prime}, m, s^{\prime}\right) \in P\right\}
$$

be the set of program states.

Definition 2 Let $P$ be a program. A triple

$$
S: \theta \rightarrow \omega, H: \theta \rightarrow \operatorname{Ord}, T: \theta \rightarrow\left({ }^{\mathrm{Ord}} 2\right)
$$

is an ordinal computation by $P$ if the following hold:

a) $\theta$ is a successor ordinal or $\theta=$ Ord; $\theta$ is the length of the computation.

b) $S(0)=H(0)=0$; the machine starts in state 0 with head position 0 . 
c) If $t<\theta$ and $S(t) \notin \operatorname{state}(P)$ then $\theta=t+1$; the machine stops if the machine state is not a program state of $P$.

d) If $t<\theta$ and $S(t) \in \operatorname{state}(P)$ then $t+1<\theta$; choose the unique command $\left(s, c, c^{\prime}, m, s^{\prime}\right) \in P$ with $S(t)=s$ and $T(t)_{H(t)}=c$; this command is executed as follows:

$$
\begin{aligned}
T(t+1)_{\xi} & =\left\{\begin{array}{l}
c^{\prime}, \text { if } \xi=H(t) ; \\
T(t)_{\xi}, \text { else; }
\end{array}\right. \\
S(t+1) & =s^{\prime} ; \\
H(t+1) & =\left\{\begin{array}{l}
H(t)+1, \text { if } m=1 ; \\
H(t)-1, \text { if } m=0 \text { and } H(t) \text { is a successor ordinal; } \\
0, \text { else. }
\end{array}\right.
\end{aligned}
$$

e) If $t<\theta$ is a limit ordinal, the machine constellation at $t$ is determined by taking inferior limits:

$$
\begin{aligned}
\forall \xi \in \operatorname{Ord} T(t)_{\xi} & =\liminf _{r \rightarrow t} T(r)_{\xi} ; \\
S(t) & =\liminf _{r \rightarrow t} S(r) ; \\
H(t) & =\liminf _{s \rightarrow t, S(s)=S(t)} H(s) .
\end{aligned}
$$

The computation is obviously recursively determined by the initial tape contents $T(0)$ and the program $P$. We call it the ordinal computation by $P$ with input $T(0)$. If the computation stops, $\theta=\beta+1$ is a successor ordinal and $T(\beta)$ is the final tape content. In this case we say that $P$ computes $T(\beta)$ from $T(0)$ and write $P: T(0) \mapsto T(\beta)$.

This interpretation of programs yields associated notions of computability.

Definition $3 A$ partial function $F$ : Ord $2 \rightarrow^{\text {Ord }} 2$ is ordinal computable if there is a program $P$ such that $P: T \mapsto F(T)$ for every $T \in \operatorname{dom}(F)$.

By coding, the notion of ordinal computability can be extended to other domains. We can e.g. code an ordinal $\delta \in$ Ord by the characteristic function $\chi_{\{\delta\}}:$ Ord $\rightarrow 2, \chi_{\{\delta\}}(\xi)=1$ iff $\xi=\delta$, and define:

Definition 4 A partial function $F:$ Ord $\rightarrow$ Ord is ordinal computable if the function $\chi_{\{\delta\}} \mapsto \chi_{\{F(\delta)\}}$ is ordinal computable.

We also consider computations involving finitely many ordinal parameters.

Definition 5 A subset $x \subseteq$ Ord is ordinal computable from finitely many ordinal parameters if there a finite subset $z \subseteq$ Ord and a program $P$ such that $P: \chi_{z} \mapsto \chi_{x}$.

In view of our intended applications of ordinal computations to models of set theory we note some absoluteness properties: 
Lemma 1 Let $(M, \in)$ be a transitive model of $\mathrm{ZF}^{-}$, i.e., of ZERMELO-FrAENKEL set theory without the powerset axiom. Let $P$ be a program and let $T(0)$ : Ord $\rightarrow 2$ be an initial tape content so that $T(0) \uparrow(\operatorname{Ord} \cap M)$ is definable in $M$. Let $S: \theta \rightarrow \omega, H: \theta \rightarrow \operatorname{Ord}, T: \theta \rightarrow\left({ }^{\mathrm{Ord}} 2\right)$ be the ordinal computation by $P$ with input $T(0)$. Then:

a) The ordinal computation by $P$ with input $T(0)$ is absolute for $(M, \in)$ below $(\operatorname{Ord} \cap M)$, i.e.,

$$
S: \theta \cap M \rightarrow \omega, H: \theta \cap M \rightarrow \operatorname{Ord}, \bar{T}: \theta \cap M \rightarrow\left({ }^{\operatorname{Ord} \cap M} 2\right)
$$

with $\bar{T}(t)=T(t) \uparrow(\operatorname{Ord} \cap M)$ is the ordinal computation by $P$ with input $T(0) \uparrow(\operatorname{Ord} \cap M)$ as computed in the model $(M, \in)$.

b) If Ord $\subseteq M$ then the ordinal computations by $P$ in $M$ and in the universe $V$ are equal.

c) Let Ord $\subseteq M$ and $x, y \subseteq$ Ord, $x, y \in M$. Then $P: \chi_{x} \mapsto \chi_{y}$ if and only if $(M, \in) \vDash " P: \chi_{x} \mapsto \chi_{y} "$.

d) Let $x, y \subseteq \operatorname{Ord}, x, y \in M$. Assume that $(M, \in) \vDash " P: \chi_{x} \mapsto \chi_{y}$ ". Then $P: \chi_{x} \mapsto \chi_{y}$.

The properties follow from the observation that the recursion in Definition 2 is clearly absolute between $M$ and $V$. Note that the converse of d) is in general false. With the subsequent results on constructibility we could let $M=L_{\delta}$ be the minimal level of the constructible hierarchy which is a model of $\mathrm{ZF}^{-}$. If $P$ is a program which searches for the minimal ordinal $\delta$ such that $L_{\delta}$ is a $\mathrm{ZF}^{-}$-model then $P$ will stop in $V$ but not in $M$.

\section{Ordinal Algorithms}

We present a number of fundamental algorithms which can be implemented as ordinal computations. Our emphasis is not on writing concrete programs as in Definition 1 but on showing that programs exist. It thus suffices to present basic ideas and algorithms together with methods to combine these into complex algorithms. We shall freely use informal "higher programming languages" to describe algorithms. Algorithms are based on data formats for the representation of input and output values. Again we shall not give detailed definitions but only indicate crucial features of the formats.

The intended computations will deal with ordinals and sequences of ordinals. The simplest way of representing the ordinal $\alpha \in$ Ord in an ordinal machine is by a tape whose content is the characteristic function of $\{\alpha\}$ :

$$
\chi_{\{\alpha\}}: \text { Ord } \rightarrow 2, \chi_{\{\alpha\}}(\xi)=1 \text { iff } \xi=\alpha .
$$

A basic task is to find or identify this ordinal $\alpha$ : initially the head is in position

0 , it then moves to the right until it stops exactly at position $\alpha$. This is achieved 
by the following program:

$$
P=\{(0,0,0,1,0),(0,1,1,1,1),(1,0,0,0,2),(1,1,1,0,2)\} .
$$

The program is in state 0 until it reads a 1 , then it goes one cell to the right, one cell to the left, and stops because 2 is not a program state. Informally the algorithm may be written as

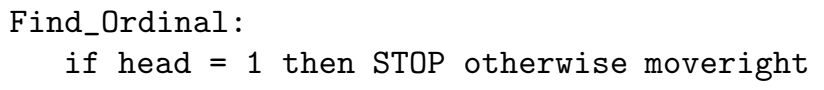

Similarly one can let the head find (the beginning) of any finite 0-1-bitstring $b_{0} \ldots b_{k-1}$ :

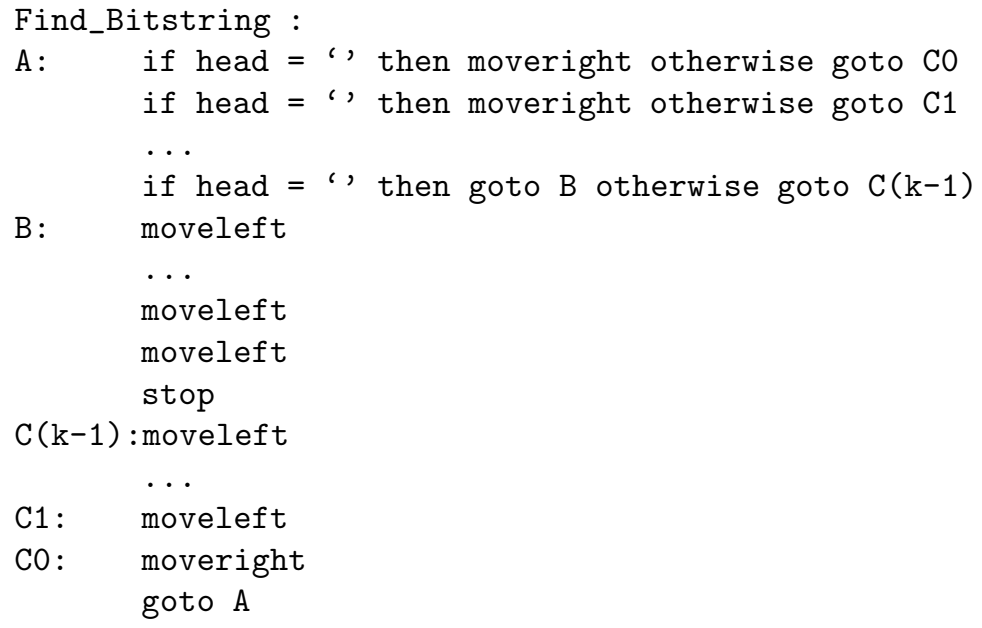

In view of this algorithm we may assume that the tape contains arbitrary symbols coded by finite bitstrings instead of single bits. Note that the above programs obviously perform the intended tasks on standard TURING machines. The limit rules are designed to lift this behaviour continuously to transfinite ordinals.

Often one has to reset the head to its initial position 0 . There are several methods to achieve this. A universal one assumes that there is a unique initial inscription start on the tape which indicates the 0-position:

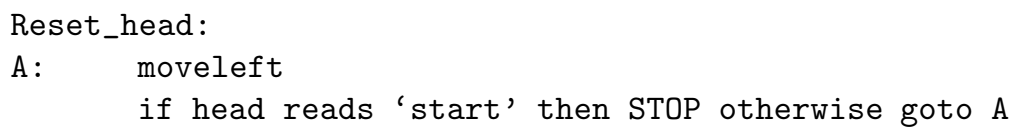

It will be convenient to work with several tapes side-by-side instead of just one. This corresponds to the idea of program variables whose values are checked and manipulated. One can simulate an $n$-tape machine on a 1 -tape machine. The contents $\left(T_{\xi}^{i} \mid \xi \in \mathrm{Ord}\right)$ of the $i$-th tape are successively written into the cells of tape $T$ indexed by ordinals $2 n \xi+2 i$ :

$$
T_{2 n \xi+2 i}=T_{\xi}^{i}
$$


The head position $H^{i}$ on the $i$-th tape is simulated by writing 1 's into an initial segment of length $H^{i}$ of cells with indices of the form $2 n \xi+2 i+1$ :

$$
T_{2 n \xi+2 i+1}=\left\{\begin{array}{l}
1, \text { if } \xi<H^{i} \\
0, \text { else }
\end{array}\right.
$$

So two tapes with contents $a_{0} a_{1} a_{2} a_{3} a_{4} \ldots$ and $b_{0} b_{1} b_{2} b_{3} b_{4} \ldots$ and head positions 3 and 1 respectively are coded as

$$
T=a_{0} 1 b_{0} 1 a_{1} 1 b_{1} 0 a_{2} 1 b_{2} 0 a_{3} 0 b_{3} 0 a_{4} 0 b_{4} 0 \ldots \ldots
$$

We describe operations of machines with several tapes by commands like moveright2 or print3 = ' ', where the number of the active tape is adjoined to the right. There are canonical but tedious translations from programs for $n$-tape machines into corresponding programs for 1-tape machines. A manipulation of the $i$-th tape amounts to first finding the head marker at ordinals of form $2 n \xi+2 i+1$; moving left by one cell one obtains the corresponding cell content for possible modification; the subsequent head movement is simulated by moving right again, writing a 0 , moving $2 n$ cells to the right or left, and printing a 1 ; if a left-movement goes across a limit ordinal, then a "1" has to be printed into cell $2 i+1$.

The subsequent algorithms will be presented as multiple tape algorithms. One can assume that one or more of the tapes serve as standard TURING tapes on which ordinary TURING recursive functions are computed. Since the usual syntactical operations for a language of set theory are intuitively computable we can assume by the CHURCH-TURING thesis that these operations are ordinal computed on some of the ordinal tapes. This will be used in the ordinal computation of the constructible model $L$.

Basic operations on ordinals are ordinal computable. Let the ordinals $\alpha$ and $\beta$ be given on tapes 0 and 1 as their characteristic functions $\chi_{\{\alpha\}}$ and $\chi_{\{\beta\}}$. The following algorithm compares the ordinals and indicates the result of the comparison by its "stopping state":

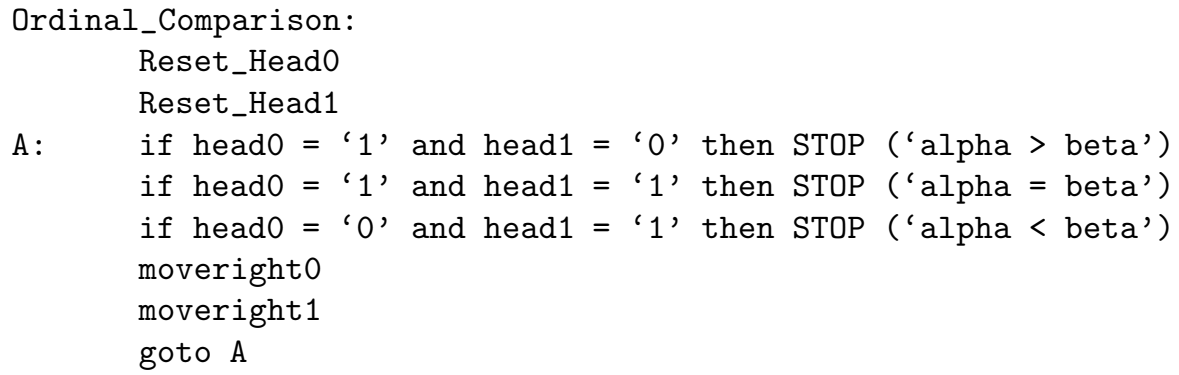

Obviously there are ordinal algorithms to reset a register containing an ordinal to 0 , or to copy one ordinal register to another one. The ordinal sum $\alpha+\beta$ and product $\alpha \cdot \beta$ are computable as follows:

Ordinal_Addition: 


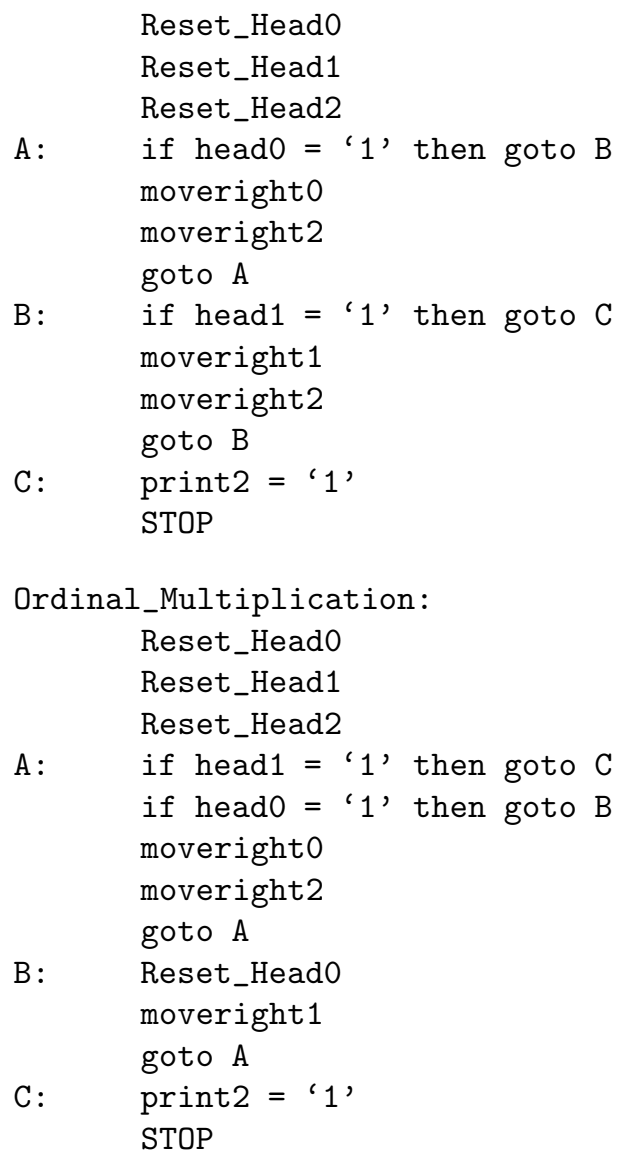

The class $\operatorname{Ord}^{<\omega}=\{s \mid \exists k<\omega s: k \rightarrow$ Ord $\}$ of finite sequences of ordinals will be of particular interest for relating ordinal computability to the iterated definability of GöDEL's constructible universe. We code a sequence $\left(\alpha_{0}, \ldots, \alpha_{k-1}\right): k \rightarrow$ Ord by a tape which starts with an initial symbol "(", followed by $k$ intervals of 0 's of lengths $\alpha_{0}, \ldots \alpha_{k-1}$ respectively, which are separated by a separation symbol "," and then a closing ")". So $(1, \omega, \omega+2)$ is coded as

$$
(0,00 \ldots, 00 \ldots 00)
$$

If the sequence is given on tape 0 and a natural number $n$ on tape 1 then the $n$-th element of the sequence can be output on tape 2 by the following algorithm:

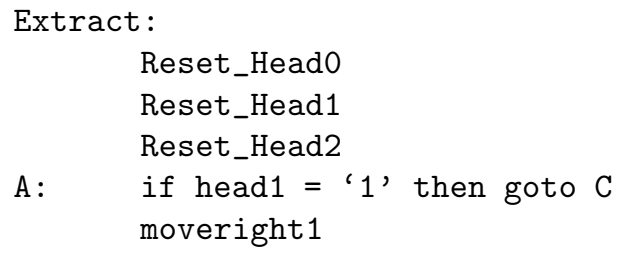




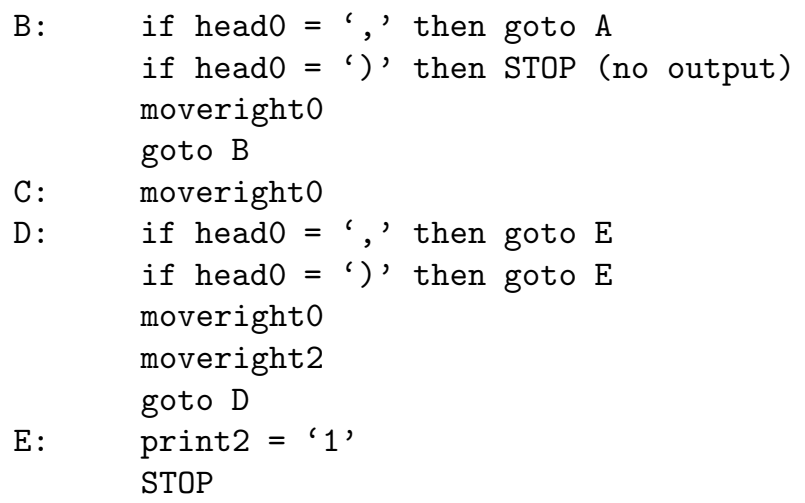

Another important operation on sequences is the replacement of the $n$-th element of a sequence $s$ of ordinals by a given ordinal $\alpha$; if the given sequence is shorter than $n+1$, it is padded by 0 's up to length $n+1$. Formally this operation on sequences is defined as $s \mapsto s \frac{\alpha}{n}$ where $\operatorname{dom}\left(s \frac{\alpha}{n}\right)=\operatorname{dom}(s) \cup(n+1)$ and

$$
s \frac{\alpha}{n}(i)=\left\{\begin{array}{l}
s(i), \text { if } i \in \operatorname{dom}(s) \backslash\{n\} \\
\alpha, \text { if } i=n \\
0, \text { else. }
\end{array}\right.
$$

Let the original sequence be given on tape 0 , the natural number $n$ on tape 1 , and the ordinal $\alpha$ on tape 2 . The modified sequence $s \frac{\alpha}{n}$ can be output on tape 3 by the following algorithm:

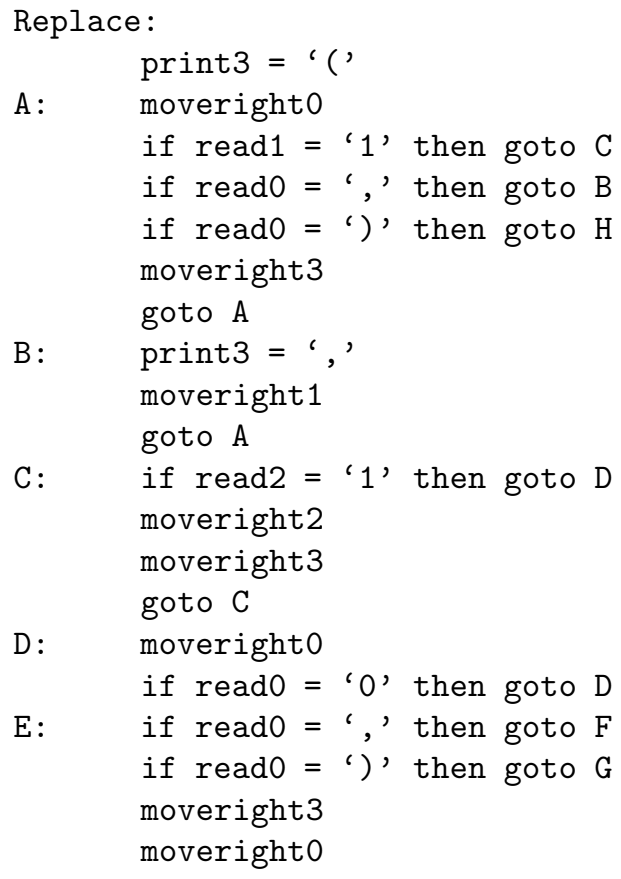




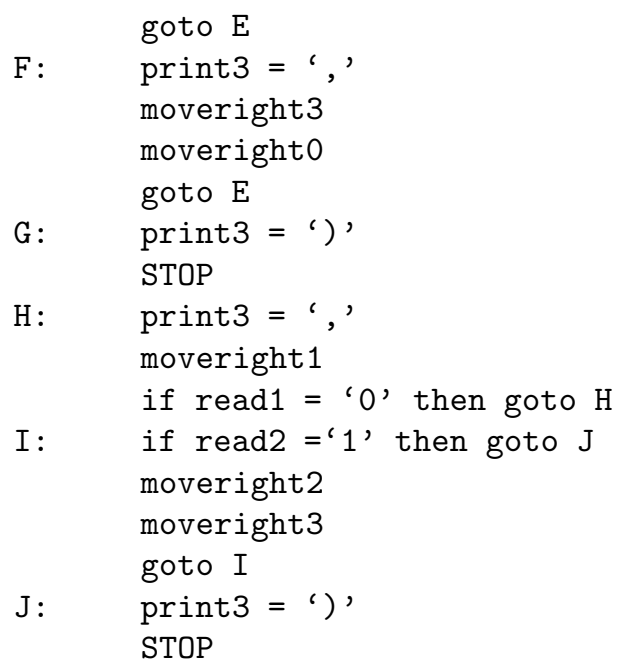

With the subroutine mechanism known from ordinary programming the basic algorithms can be combined into complex algorithms for comparing and manipulating ordinal sequences. We can, e.g., carry out a syntactic manipulation on a standard TURING tape which outputs requests for checking or manipulating elements of ordinal sequences. According to the requests the appropriate elements can be extracted and subjected to some algorithms whose results can be substituted into the original sequences.

\section{Enumerating Finite Sequences of Ordinals}

For $X$ a class let $[X]^{<\omega}=\{z \subseteq X \mid z$ is finite $\}$ and $X<\omega=\{s \mid \exists k<\omega s: k \rightarrow$ $X\}$ be the class of all finite subsets of $X$ and of all finite sequences from $X$ respectively.

Finite sequences of ordinals are finite sets of ordered pairs:

$$
\operatorname{Ord}^{<\omega} \subseteq[\omega \times \mathrm{Ord}]^{<\omega}
$$

Well-order $\omega \times$ Ord by

$$
(m, \alpha) \prec(n, \beta) \text { iff } \alpha<\beta \text { or }(\alpha=\beta \wedge m<n) .
$$

Define a canonical well-order $\left([\omega \times \text { Ord }]^{<\omega}, \prec^{*}\right)$ by largest difference:

$$
s \prec^{*} s^{\prime} \text { iff } \exists x \in s^{\prime} \backslash s\{y \in s \mid y \succ x\}=\left\{y \in s^{\prime} \mid y \succ x\right\} .
$$

One can show inductively that $\left([Y]^{<\omega}, \prec^{*}\right)$ is a well-order on initial segments $Y$ of $(\omega \times$ Ord, $\prec)$. So $\prec^{*}$ well-orders $[\omega \times \text { Ord }]^{<\omega}$ and hence Ord ${ }^{<\omega}$. We note an important substitution property of the well-order:

Lemma 2 If $s, t \in \operatorname{Ord}^{<\omega}, m \in \operatorname{dom}(s), \operatorname{dom}(s) \subseteq \operatorname{dom}(t), s \uparrow(\operatorname{dom}(s) \backslash$ $\{m\})=t \uparrow(\operatorname{dom}(s) \backslash\{m\}), t(m)<s(m), \forall i \in \operatorname{dom}(t) \backslash \operatorname{dom}(s) t(i)<s(m)$ then

$$
t \prec * s \text {. }
$$


So replacing an arbitrary ordinal $s(m)$ of $s$ by possibly many smaller ordinals leads to a descent in $\prec^{*}$. The substitution property will correspond to the substitution of a bounded variable below some bound by terms with parameters smaller than that bound. This will lead to a recursive definition of bounded truth in $L$ along the $\prec^{*}$-relation.

We define an enumeration $S:$ Ord $\rightarrow \operatorname{Ord}^{<\omega}$ (with repetitions) of $\mathrm{Ord}^{<\omega}$ which is compatible with $\prec^{*}$ and which can be computed by an ordinal machine. The idea of the construction is to recursively apply the replacement operation $s \frac{\alpha}{n}$ to sequences $s$ which have been enumerated before.

For $(m, \alpha) \in \omega \times$ Ord define functions $S_{m \alpha}: \theta_{m \alpha} \rightarrow \operatorname{Ord}^{<\omega}$ such that for $(m, \alpha) \prec(n, \beta), S_{m \alpha}$ is an initial segment of $S_{n \beta}$. Set $S_{00}: 1 \rightarrow \operatorname{Ord}^{<\omega}$, $S_{00}(0)=\emptyset$. For $\beta>0$ set

$$
S_{0 \beta}=\bigcup_{(m, \alpha) \prec(0, \beta)} S_{m \alpha} .
$$

Assume that $S_{m \alpha}: \theta_{m \alpha} \rightarrow \operatorname{Ord}^{<\omega}$ is defined. Then define $S_{m+1, \alpha}: \theta_{m \alpha} \cdot 2 \rightarrow$ Ord ${ }^{<\omega}$ by: $S_{m+1, \alpha}\left\lceil\theta_{m \alpha}=S_{m \alpha}\right.$; for $\xi<\theta_{m \alpha}$ let

$$
S_{m+1, \alpha}\left(\theta_{m \alpha}+\xi\right)=S_{m \alpha}(\xi) \frac{\alpha}{m} .
$$

Finally set

$$
S=\bigcup_{(m, \alpha) \in \omega \times \text { Ord }} S_{m \alpha}
$$

Lemma 3 a) $S:$ Ord $\rightarrow \operatorname{Ord}^{<\omega}$ is a surjection.

b) If $\xi<\zeta$ then $S(\xi)=S(\zeta)$ or $S(\xi) \prec * S(\zeta)$.

Proof a) We show by induction on $\alpha$ that $S_{0 \alpha}: \theta_{0 \alpha} \rightarrow \alpha^{<\omega}$ is a surjection. The initial case $\alpha=0$ and the limit step are easy. Consider $\alpha=\beta+1$ and some $s \in \alpha^{<\omega}, s: k \rightarrow \alpha$. Let $\bar{s}: k \rightarrow \alpha$ be the following restriction of $s$ to $\beta$ :

$$
\bar{s}(i)=\left\{\begin{array}{l}
s(i), \text { if } s(i)<\beta \\
0, \text { if } s(i)=\beta
\end{array}\right.
$$

By the inductive assumption there is $\xi<\theta_{0 \beta}$ such that $S_{0 \beta}(\xi)=\bar{s}$. Then

$$
S_{0 \alpha}\left(\theta_{0 \beta} \cdot\left(\sum_{i<k, s(i)=\beta} 2^{i}\right)+\xi\right)=s .
$$

b) follows from the substitution property.

The enumeration $S$ of $\operatorname{Ord}^{<\omega}$ is ordinal computable using coding methods from the previous paragraph. We indicate a program which writes the values of $S$ consecutively on a tape:

$$
S(0) S(1) S(2) \ldots S(\omega) S(\omega+1) \ldots S(\omega+\omega) \ldots S(\alpha) \ldots \ldots
$$


where each $S(\alpha)$ is of the form

$$
(0 \ldots 0,0 \ldots 0, \ldots \ldots, 0 \ldots 0)
$$

The algorithm is based on the Replace-algorithm from the previous section:

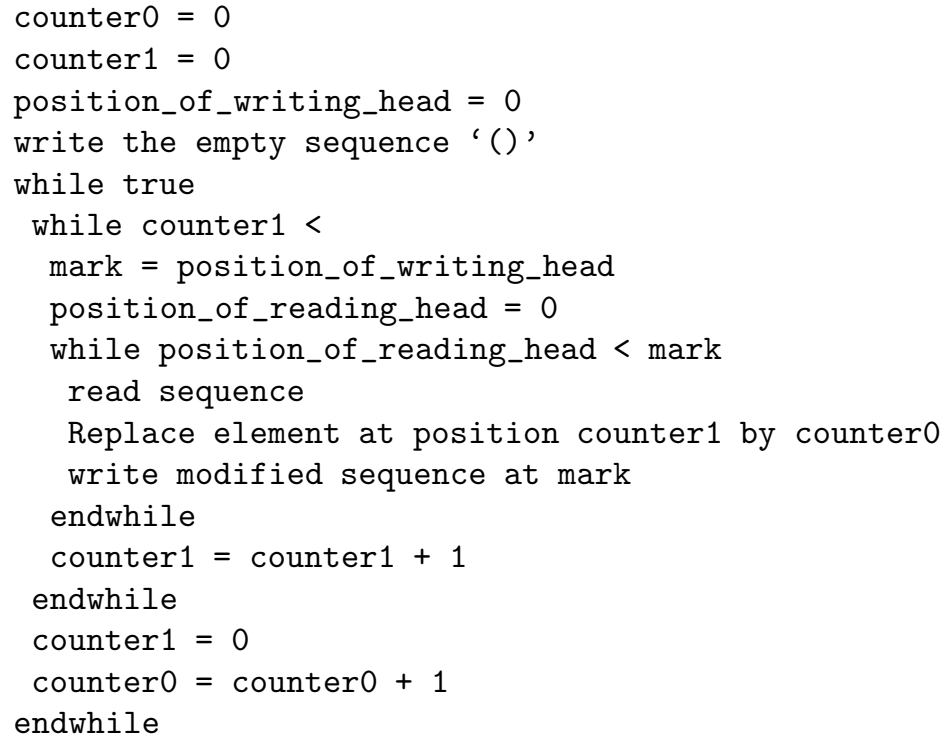

The procedure will eventually be extended as to write a bounded truth function for the constructible hierarchy.

\section{The Constructible Hierarchy}

KURT GöDEL [3] defined the inner model $L$ of constructible sets as the union of a hierarchy of levels $L_{\alpha}$ :

$$
L=\bigcup_{\alpha \in \text { Ord }} L_{\alpha}
$$

where the hierarchy is defined by: $L_{0}=\emptyset, L_{\delta}=\bigcup_{\alpha<\delta} L_{\alpha}$ for limit ordinals $\delta$, and $L_{\alpha+1}=$ the set of all sets which are first-order definable in the structure $\left(L_{\alpha}, \in\right)$. The standard reference to the theory of the model $L$ is the book [2] by Keith Devlin.

An element of $L$ is definable over some $L_{\alpha}$ from parameters which are themselves definable over some $L_{\beta}, \beta<\alpha$ in some other parameters and so forth. We therefore introduce a language with definable terms, which in turn may involve definable terms etc.

Consider a language with symbols $(),,\{\},, \mid, \in,=, \wedge, \neg, \forall, \exists$ and variables $v_{0}, v_{1}, \ldots$. We define (bounded) formulas and (bounded) terms by a common recursion on the lenghts of words formed from these symbols:

- the variables $v_{0}, v_{1}, \ldots$ are terms; 
- if $s$ and $t$ are terms then $s=t$ and $s \in t$ are formulas;

- if $\varphi$ and $\psi$ are formulas then $\neg \varphi,(\varphi \wedge \psi), \forall v_{i} \in v_{j} \varphi$ and $\exists v_{i} \in v_{j} \varphi$ are formulas;

- if $\varphi$ is a formula then $\left\{v_{i} \in v_{j} \mid \varphi\right\}$ is a term.

For terms and formulas of this language define free and bound variables:

- free $\left(v_{i}\right)=\left\{v_{i}\right\}$, bound $\left(v_{i}\right)=\emptyset$;

$-\operatorname{free}(s=t)=\operatorname{free}(s \in t)=\operatorname{free}(s) \cup \operatorname{free}(t) ;$

$-\operatorname{bound}(s=t)=\operatorname{bound}(s \in t)=\operatorname{bound}(s) \cup \operatorname{bound}(t)$;

- $\operatorname{free}(\neg \varphi)=\operatorname{free}(\varphi), \operatorname{bound}(\neg \varphi)=\operatorname{bound}(\varphi)$;

- free $((\varphi \wedge \psi))=\operatorname{free}(\varphi) \cup$ free $(\psi)$, bound $((\varphi \wedge \psi))=\operatorname{bound}(\varphi) \cup \operatorname{bound}(\psi)$;

- free $\left(\forall v_{i} \in v_{j} \varphi\right)=\operatorname{free}\left(\exists v_{i} \in v_{j} \varphi\right)=\operatorname{free}\left(\left\{v_{i} \in v_{j} \mid \varphi\right\}\right)=\left(\operatorname{free}(\varphi) \cup\left\{v_{j}\right\}\right) \backslash$ $\left\{v_{i}\right\}$

- $\operatorname{bound}\left(\forall v_{i} \in v_{j} \varphi\right)=\operatorname{bound}\left(\exists v_{i} \in v_{j} \varphi\right)=\operatorname{bound}\left(\left\{v_{i} \in v_{j} \mid \varphi\right\}\right)=$ $=\operatorname{bound}(\varphi) \cup\left\{v_{i}\right\}$.

For technical reasons we will be interested in terms and formulas in which

- no bound variable occurs free,

- every free variable occurs exactly once.

Such terms and formulas are called tidy; with tidy formulas one avoids having to deal with the interpretation of one free variable at different positions within a formula.

In recursive truth definitions one reduces the truth of formulas to the truth of simpler formulas. The term complexity $\operatorname{tc}(t)$ and $\operatorname{tc}(\varphi)$ of terms and formulas is defined recursively:

$-\operatorname{tc}\left(v_{i}\right)=0$

$-\operatorname{tc}(s=t)=\operatorname{tc}(s \in t)=\max (\operatorname{tc}(s), \operatorname{tc}(t))$;

$-\operatorname{tc}(\neg \varphi)=\operatorname{tc}\left(\forall v_{i} \in v_{j} \varphi\right)=\operatorname{tc}\left(\exists v_{i} \in v_{j} \varphi\right)=\operatorname{tc}(\varphi) ;$

$-\operatorname{tc}(\varphi \wedge \psi)=\max (\operatorname{tc}(\varphi), \operatorname{tc}(\psi))$;

$-\operatorname{tc}\left(\left\{v_{i} \in v_{j} \mid \varphi\right\}\right)=\operatorname{tc}(\varphi)+1$.

We can define a pre-wellordering $<_{\text {Form }}$ of the set of all bounded formulas by

$\varphi<$ Form $\psi$ iff $\operatorname{tc}(\varphi)<\operatorname{tc}(\psi)$ or $(\operatorname{tc}(\varphi)=\operatorname{tc}(\psi) \wedge$ length $(\varphi)<\operatorname{length}(\psi))$. 
Obviously the syntactical notions and operations of this language are TURING computable and therefore ordinal computable. Also there is an ordinal computable enumeration of all formulas which is compatible with $<_{\text {Form }}$.

An assignment for a term $t$ or formula $\varphi$ is a finite sequence $a: k \rightarrow V$ so that for every free variable $v_{i}$ of $t$ or $\varphi$ we have $i<k$; $a(i)$ will be the interpretation of $v_{i}$. The value of $t$ or the truth value of $\varphi$ is determined by the assignment $a$. We write $t[a]$ and $\varphi[a]$ for the values of $t$ und $\varphi$ under the assignment $a$.

Concerning the constructible hierarchy $L$, it is shown by an easy induction on $\alpha$ that every element of $L_{\alpha}$ is the interpretation $t\left[\left(L_{\alpha_{0}}, L_{\alpha_{1}}, \ldots, L_{\alpha_{k-1}}\right)\right]$ of some tidy term $t$ with an assignment $\left(L_{\alpha_{0}}, L_{\alpha_{1}}, \ldots, L_{\alpha_{k-1}}\right)$ whose values are constructible levels $L_{\alpha_{i}}$ with $\alpha_{0}, \ldots, \alpha_{k-1}<\alpha$. This will allow to reduce bounded quantifications $\forall v \in L_{\alpha}$ or $\exists v \in L_{\alpha}$ to the substitution of terms of lesser complexity. Moreover, the truth of (bounded) formulas in $L$ is captured by tidy bounded formulas of the form $\varphi\left[\left(L_{\alpha_{0}}, L_{\alpha_{1}}, \ldots, L_{\alpha_{k-1}}\right)\right]$.

We shall code an assignment of the form $\left(L_{\alpha_{0}}, L_{\alpha_{1}}, \ldots, L_{\alpha_{k-1}}\right)$ by its sequence of ordinal indices, i.e., we write

$$
t\left[\left(\alpha_{0}, \alpha_{1}, \ldots, \alpha_{k-1}\right)\right] \text { or } \varphi\left[\left(\alpha_{0}, \alpha_{1}, \ldots, \alpha_{k-1}\right)\right]
$$

instead of

$$
t\left[\left(L_{\alpha_{0}}, L_{\alpha_{1}}, \ldots, L_{\alpha_{k-1}}\right)\right] \text { or } \varphi\left[\left(L_{\alpha_{0}}, L_{\alpha_{1}}, \ldots, L_{\alpha_{k-1}}\right)\right] .
$$

The relevant assignments are thus elements of $\mathrm{Ord}^{<\omega}$ and can be handled by the programs of the previous section. Since the bounded language is recursive we can modify the enumeration program so that all assigned tidy formulas $\varphi\left[\left(\alpha_{0}, \alpha_{1}, \ldots, \alpha_{k-1}\right)\right]$ occur in the enumeration: for a fixed assigment $a=\left(\alpha_{0}, \alpha_{1}, \ldots, \alpha_{k-1}\right)$ list the pairs $(a, \varphi)$ where $\varphi$ is a tidy formula with free $(\varphi) \subseteq k$ in an order compatible with $<_{\text {Form }}$. The following is a straightforward extension of the enumeration program of the previous section:

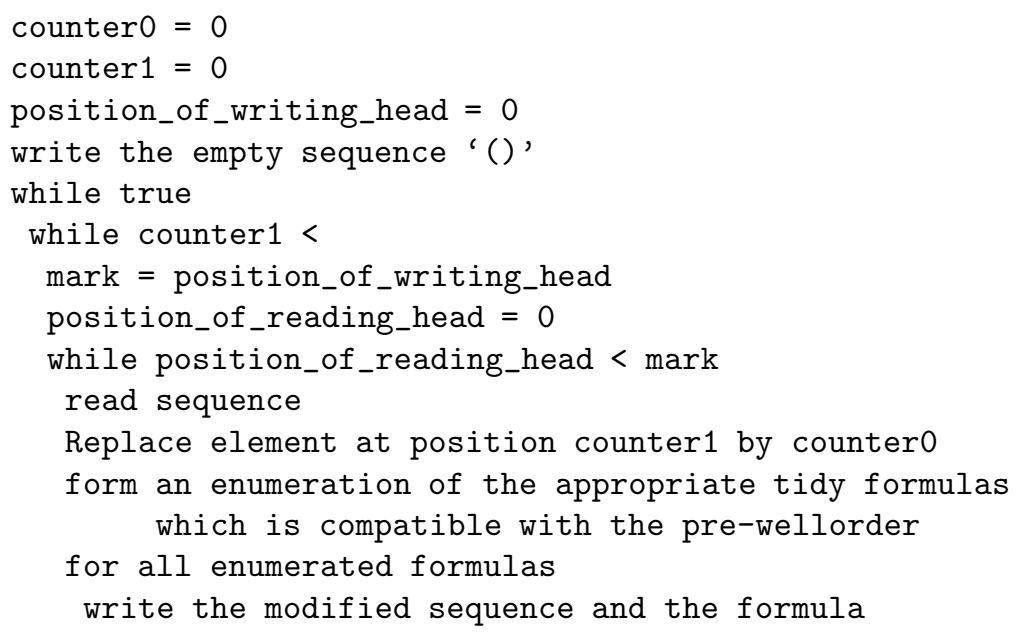




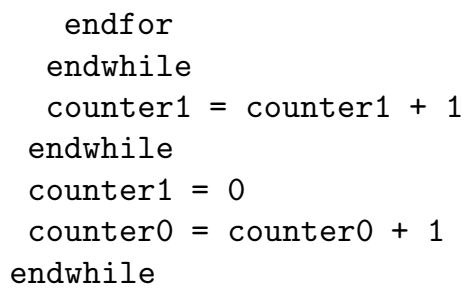

\section{$6 \quad$ A Bounded Truth Function for $L$}

We define a bounded truth function $W$ for the constructible hierarchy on the class

$$
A=\left\{(a, \varphi) \mid a \in \operatorname{Ord}^{<\omega}, \varphi \text { is a tidy bounded formula, free }(\varphi) \subseteq \operatorname{dom}(a)\right\}
$$

of all "tidy pairs" of assignments and formulas. Define the bounded constructible truth function $W: A \rightarrow 2$ by

$$
W(a, \varphi)=1 \text { iff } \varphi[a] .
$$

The function $W$ has a recursive definition along the enumeration of $A$ given by the enumeration algorithm from the preceding section. We explain the principal idea of the recursion with a bounded quantification like $\exists v_{i} \in v_{j} \varphi[a]$. If $a(j)=\alpha$ then the assigned formula is satisfied if and only if there is a witness for $\varphi$ in $L_{\alpha}$. By the recursive definition of $L_{\alpha}$ such a witness must be the interpretation $t[b]$ of a term where $b(l)<\alpha$ for every free variable $v_{l}$ of $t$. If one chooses $t$ such that it has no variable in common with $\varphi$ the assignment $b$ can be taken to further satisfy $b(j)<a(j)=\alpha$. The substitution property of Lemma 2] leads to the evaluation of $\varphi \frac{t}{v_{i}}[b]$ for some $b \prec^{*} a$ which is the basis for the subsequent recursion.

As we want to work with tidy formulas a technical problem has to be solved. The variable $v_{i}$ might occur in $\varphi$ in several places which renders the straightforward substitution $\varphi \frac{t}{v_{i}}$ "untidy". We "tidy up" $\varphi \frac{t}{v_{i}}$ by renaming variables. The assignment of the variables of $t$ has to be modified accordingly.

So consider a formula $\varphi$, a variable $v_{i}$, a term $t$, and an assignment $a$ with $\left\{i \mid v_{i} \in\right.$ free $\left.(\varphi)\right\} \cup\left\{v_{j}\right\} \subseteq \operatorname{dom}(a)$, where $v_{j}$ is a further variable thought to be a bound for $t$ as in $\exists v_{i} \in v_{j} \varphi[a]$. Also assume that $\varphi$ and $t$ have no common variable and do not contain $v_{j}$. Define the tidy substitution $\left(\varphi \frac{t}{v_{i}}\right)^{\text {tidy }}$ of $v_{i}$ by $t$ into $\varphi$ as follows. If $v_{i} \notin$ free $(\varphi)$ then let $\left(\varphi \frac{t}{v_{i}}\right)^{\text {tidy }}=\varphi$. Otherwise ensure that $v_{i}$ is not a bound variable of $\varphi$ by possibly renaming bound variables. Let $\varphi^{\prime}$ be the renamed formula. Then rename all occurances of $v_{i}$ in $\varphi^{\prime}$ by pairwise different new variables $w_{0}, \ldots, w_{k-1}$, say. Obtain terms $t_{0}, \ldots, t_{k-1}$ from the given term $t$ by renaming all variables with new variables so that for $i \neq j$ the terms $t_{i}$ and $t_{j}$ do not have common variables; call $t_{0}, \ldots, t_{k-1}$ copies of $t$. Now set

$$
\left(\varphi \frac{t}{v_{i}}\right)^{\mathrm{tidy}}=\varphi^{\prime} \frac{t_{0} \ldots t_{k-1}}{w_{0} \ldots w_{k-1}} .
$$


The assignment $a$ has to be extended to an assignment $b \prec^{*} a$ in line with the various renaming operations.

We define that the assignment $b$ adequately extends $a$ for the tidy substitution $\left(\varphi \frac{t}{v_{i}}\right)^{\text {tidy }}$ bounded by $v_{j}$ if

a) $\forall l \in \operatorname{dom}(a) \backslash\{j\} b(l)=a(l)$ and $b(j)<a(j)$;

b) $\forall l \in \operatorname{dom}(b) \backslash \operatorname{dom}(a) b(l)<a(j)$;

c) if $v_{l}$ is a variable of $t$ and $v_{l^{\prime}}$ and $v_{l^{\prime \prime}}$ are the renamings of $v_{l}$ in the copies $t_{i}$ and $t_{j}$ resp. then $b\left(l^{\prime}\right)=b\left(l^{\prime \prime}\right)$.

Note that $b$ satisfies $b \prec^{*} a$ by the substitution property Lemma 2

With these preparations we can now carry out a recursive definition of the bounded constructible truth function:

- $W(a, \neg \varphi)=1$ iff $W(a, \varphi)=0 ;$

- $W(a,(\varphi \wedge \psi))=1$ iff $W(a, \varphi)=1$ and $W(a, \psi)=1$;

- $W\left(a, \forall v_{i} \in v_{j} \varphi\right)=1$ iff for all terms $t$ and all assignments $b$ which are adequate for the tidy substitution $\left(\varphi \frac{t}{v_{i}}\right)^{\text {tidy }}$ bounded by $v_{j}$

holds $W\left(b,\left(\varphi \frac{t}{v_{i}}\right)^{\text {tidy }}\right)=1$;

- $W\left(a, \exists v_{i} \in v_{j} \varphi\right)=1$ iff there is a term $t$ and an assignment $b$ which is adequate for the tidy substitution $\left(\varphi \frac{t}{v_{i}}\right)^{\text {tidy }}$ bounded by $v_{j}$ so that $W\left(b,\left(\varphi \frac{t}{v_{i}}\right)^{\mathrm{tidy}}\right)=1$

- $W\left(a, v_{i} \in v_{j}\right)=1$ iff $a(i)<a(j)$;

- $W\left(a, v_{i} \in\left\{v_{j} \in v_{k} \mid \varphi\right\}\right)=1$ iff $W\left(a, \exists v_{j} \in v_{k}\left(v_{i}=v_{j} \wedge \varphi\right)\right)=1$;

- W(a, $\left.\left\{v_{i} \in v_{j} \mid \varphi\right\} \in v_{k}\right)=1$ iff there is a term $t$ and an assignment $b$ which is adequate for the tidy substitution $\left(\left(\left\{v_{i} \in v_{j} \mid \varphi\right\}=v_{l}\right) \frac{t}{v_{l}}\right)^{\text {tidy }}$ bounded by $v_{k}$ so that $W\left(b,\left(\left(\left\{v_{i} \in v_{j} \mid \varphi\right\}=v_{l}\right) \frac{t}{v_{l}}\right)^{\text {tidy }}\right)=1$;

- $W\left(a,\left\{v_{i} \in v_{j} \mid \varphi\right\} \in\left\{v_{m} \in v_{n} \mid \psi\right\}\right)=1$ iff there is a term $t$ and an assignment $b$ which is adequate for the tidy substitution $\left(\left(\left\{v_{i} \in v_{j} \mid \varphi\right\}=v_{m} \wedge\right.\right.$ $\left.\psi) \frac{t}{v_{m}}\right)^{\text {tidy }}$ bounded by $v_{n}$ so that $W\left(b,\left(\left(\left\{v_{i} \in v_{j} \mid \varphi\right\}=v_{m} \wedge \psi\right) \frac{t}{v_{m}}\right)^{\text {tidy }}\right)=$ 1

- $W\left(a, v_{i}=v_{j}\right)=1$ iff $a(i)=a(j) ;$

- $W\left(a, v_{i}=\left\{v_{j} \in v_{k} \mid \varphi\right\}\right)=1$ iff

$W\left(a, \forall v_{l} \in v_{i} \exists v_{j} \in v_{k}\left(\varphi \wedge v_{l}=v_{j}\right)\right)=1$ and

$W\left(a, \forall v_{j} \in v_{k}\left(\varphi \rightarrow \exists v_{l} \in v_{i} v_{l}=v_{j}\right)\right)=1$;

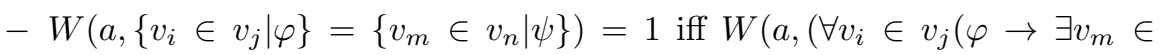
$\left.\left.v_{n}\left(v_{i}=v_{m} \wedge \psi\right)\right)\right)=1$ and $\left.W\left(a, \forall v_{m} \in v_{n}\left(\psi \rightarrow \exists v_{i} \in v_{j}\left(v_{m}=v_{i} \wedge \varphi\right)\right)\right)\right)=$ 1. 
In all clauses the determination of $W(a, \varphi)$ is reduced to values $W(b, \psi)$. where the relevant arguments $(b, \psi)$ are of lesser complexity than $(a, \varphi)$ : either the assignments satisfy $b \prec^{*} a$ or we have that $a=b$ and $\psi<$ Form $\varphi$. Therefore $W$ has a recursive definition along the enumeration given by the algorithm of the preceding section.

The programming techniques introduced above allow to incorporate the recursive definition of $W$ into the enumeration algorithm for the class $A$ of admissible pairs. This leads to our main results:

Lemma 4 The bounded truth function $W$ for the constructible universe is ordinal computable.

Theorem 2 A set $x$ of ordinals is ordinal computable from a finite set of ordinal parameters if and only if it is an element of the constructible universe $L$.

Proof Let $x \subseteq$ Ord be ordinal computable by the program $P$ from the finite set $\left\{\alpha_{0}, \ldots, \alpha_{k-1}\right\}$ of ordinal parameters: $P: \chi_{\left\{\alpha_{0}, \ldots, \alpha_{k-1}\right\}} \mapsto \chi_{x}$. By Lemma 11 c) the same computation can be carried out inside the inner model $L$ :

$$
(L, \in) \vDash P: \chi_{\left\{\alpha_{0}, \ldots, \alpha_{k-1}\right\}} \mapsto \chi_{x} .
$$

Hence $\chi_{X} \in L$ and $x \in L$.

Conversely consider $x \in L$. Choose a tidy term $t$ and an assignment $\left(\alpha_{0}, \ldots, \alpha_{k-1}\right) \in \operatorname{Ord}^{<\omega}$ such that $x=t\left[\left(\alpha_{0}, \ldots, \alpha_{k-1}\right)\right]$. An ordinal $\beta$ can be represented as

$$
\begin{aligned}
\beta & =\left\{\alpha \in L_{\beta} \mid \alpha \text { is an ordinal }\right\} \\
& =\left\{v_{k+1} \in v_{k} \mid v_{k+1} \text { is an ordinal }\right\}\left[\left(\alpha_{0}, \ldots, \alpha_{k-1}, \beta\right)\right] .
\end{aligned}
$$

Thus

$$
\begin{aligned}
\chi_{x}(\beta)=1 & \leftrightarrow \beta \in t\left[\left(\alpha_{0}, \ldots, \alpha_{k-1}\right)\right] \\
& \leftrightarrow\left(\left\{v_{k+1} \in v_{k} \mid v_{k+1} \text { is an ordinal }\right\} \in t\right)\left[\left(\alpha_{0}, \ldots, \alpha_{k-1}, \beta\right)\right] \\
& \leftrightarrow W\left(\left(\alpha_{0}, \ldots, \alpha_{k-1}, \beta\right),\left\{v_{k+1} \in v_{k} \mid v_{k+1} \text { is an ordinal }\right\} \in t\right)=1 .
\end{aligned}
$$

Using the enumeration algorithm for the truth function $W$ one can turn this equivalence into an ordinal algorithm which sends $\chi_{\left\{\alpha_{0}, \ldots, \alpha_{k-1}\right\}}$ to $\chi_{x}$. Hence $x$ is ordinal computable from the parameters $\alpha_{0}, \ldots, \alpha_{k-1} \in$ Ord.

\section{The Generalized Continuum Hypothesis in $L$}

Ordinal computability allows to reprove some basic facts about the constructible universe $L$. The analogue of the axiom of constructibility, $V=L$, is the statement that every set of of ordinals is ordinal computable from a finite set of ordinals. 
Theorem 3 The constructible model $(L, \in)$ satisfies that every set of ordinals is ordinal computable from a finite set of ordinals.

Proof Let $x \in L, x \subseteq$ Ord. By Theorem 2] take a program $P$ and a finite set $\left\{\alpha_{0}, \ldots, \alpha_{k-1}\right\}$ of ordinal parameters such that $P: \chi_{\left\{\alpha_{0}, \ldots, \alpha_{k-1}\right\}} \mapsto \chi_{x}$. By Lemma 1 c) the same computation can be carried out inside the inner model $L$ :

$$
(L, \in) \vDash P: \chi_{\left\{\alpha_{0}, \ldots, \alpha_{k-1}\right\}} \mapsto \chi_{x} .
$$

So in $L, x$ is ordinal computable from the set $\left\{\alpha_{0}, \ldots, \alpha_{k-1}\right\}$.

The following therem is proved by a condensation argument for ordinal computations which is a simple analogue of the usual condensation arguments for the constructible hierarchy.

Theorem 4 Assume that every set of ordinals is ordinal computable from a finite set of ordinals. Then:

a) Let $\kappa \geqslant \omega$ be an infinite ordinal and $x \subseteq \kappa$. Then there are ordinals $\alpha_{0}, \ldots, \alpha_{k-1}<\kappa^{+}$such that $x$ is ordinal computable from the set $\left\{\alpha_{0}, \ldots, \alpha_{k-1}\right\}$.

b) Let $\kappa \geqslant \omega$ be infinite. Then $\operatorname{card}(\mathcal{P}(\kappa))=\kappa^{+}$.

c) The generalized continuum hypothesis GCH holds.

Proof a) Take a program $P$ and a finite set $\left\{\alpha_{0}^{\prime}, \ldots, \alpha_{k-1}^{\prime}\right\}$ of ordinal parameters such that $P: \chi_{\left\{\alpha_{0}^{\prime}, \ldots, \alpha_{k-1}^{\prime}\right\}} \mapsto \chi_{x}$; let $\theta$ be the length of this ordinal computation. Take a transitive $\mathrm{ZF}^{-}$-model $(M, \in)$ such that $\alpha_{0}^{\prime}, \ldots, \alpha_{k-1}^{\prime}, \theta, \kappa, x \in M$. By Lemma \a), $(M, \in)$ also satisfies that $P: \chi_{\left\{\alpha_{0}^{\prime}, \ldots, \alpha_{k-1}^{\prime}\right\}} \mapsto \chi_{x}$. The downward LöWENHEIM-SKOLEM theorem and the Mostowski isomorphism theorem yield an elementary embedding

$$
\pi:(\bar{M}, \in) \rightarrow(M, \in)
$$

such that $\bar{M}$ is transitive, $\operatorname{card}(\bar{M})=\kappa$ and $\left\{\alpha_{0}^{\prime}, \ldots, \alpha_{k-1}^{\prime}, \theta, \kappa, x\right\} \cup \kappa \subseteq \pi^{\prime \prime} \bar{M}$. Let $\pi\left(\alpha_{0}\right)=\alpha_{0}^{\prime}, \ldots, \pi\left(\alpha_{k-1}^{\prime}\right)=\alpha_{k-1}$. Then $\alpha_{0}, \ldots, \alpha_{k-1}<\kappa^{+} \operatorname{since} \operatorname{card}(\bar{M})<$ $\kappa^{+}$. Observe that $\pi(x)=x$. Since $\pi$ is elementary $(\bar{M}, \in)$ satisfies that $P$ : $\chi_{\left\{\alpha_{0}, \ldots, \alpha_{k-1}^{\prime}\right\}} \mapsto \chi_{x}$. By Lemma 1d), $P: \chi_{\left\{\alpha_{0}, \ldots, \alpha_{k-1}^{\prime}\right\}} \mapsto \chi_{x}$ in $V$. Thus $x$ is ordinal computable from the set $\left\{\alpha_{0}, \ldots, \alpha_{k-1}\right\}$ as required.

b) follows from a) since there are a countable many programs and $\kappa^{+}$many finite sets of ordinals $<\kappa^{+}$.

c) is immediate from b)

These two theorems immediately imply GöDEL's result:

Theorem $5(L, \in) \vDash \mathrm{GCH}$. 
Other condensation arguments like the proof of the combinatorial principle $\diamond$ in $L$ can also be translated into the setting of ordinal computability in a straightforward way. It remains to be seen whether arguments involving JENSEN's fine structure theory of the constructible hierarchy [5] can be carried out with ordinal computability. One would hope that the simple concept of ordinal computation allows clear proofs of principles like $\square$ and morasses without definability complications.

\section{References}

[1] J. Richard Büchi. Weak second-order arithmetic and finite automata. Z. Math. Logik Grundlagen Math., 6:66-92, 1960.

[2] Keith Devlin. Constructibility. Perspectives in Mathematical Logic. Springer-Verlag, Berlin, 1984.

[3] Kurt Gödel. The Consistency of the Continuum Hypothesis, volume 3 of Ann. of Math. Studies. Princeton University Press, Princeton, 1940.

[4] Joel David Hamkins and Andy Lewis. Infinite Time Turing Machines. J. Symbolic Logic, 65(2):567-604, 2000.

[5] Ronald R. Jensen. The fine structure of the constructible hierarchy. Annals of Mathematical Logic, 4:229-308, 1972.

[6] Gerald E. Sacks. Higher Recursion Theory. Perspectives in Mathematical Logic. Springer-Verlag, Berlin Heidelberg, 1990. 